Apidologie, 1984, 15 (2), 137-144

\title{
BESTIMMUNG EINER POLLENFORM DER TRIFOLIUM-GRUPPE IN HONIGEN AUS ISRAEL
}

\author{
A. MAURIZIO
}

Liebefeld, Schweiz

\section{ZUSAMMENFASSUNG}

Honig aus Israel, besonders sog. «wild flowers honey» enthält oft eine Trifolium-Art als Leitoder Begleitpollen, deren Identifizierung zunächst auf gewisse Schwierigkeiten stieß. Eine vergleichende Prüfung des Pollens von 14 in Israel vorkommenden Trifolium-Arten und von 5 Varietäten von Trifolium alexandrinum erlaubte eine nähere Bestimmung dieser Form. Es handelt sich um Trifolium alexandrinum L., eine in Israel weitverbreitete Art, die in mehreren Varietäten gezüchtet wird. Das Pollenspektrum vieler Honige aus Israel nähert sich dem ägyptischer Honige, in welchen Trifolium alexandrinum ebenfalls häufig als Leitpollen zu finden ist.

\section{EINLEITUNG UND FRAGESTELLUNG}

In einer vorläufigen Mitteilung über das Pollenbild von Honigen aus Israel sind mehrere Leguminosenpollen angeführt, darunter Trifolium repens und eine an Trifolium pratense erinnernde Form, deren Bestimmung zunächst auf gewisse Schwierigkeiten stieß (Maurizıo, 1968). Da diese Form in $70 \%$ der über 100 untersuchten Israel-Honige vorkommt und, besonders im sog. "wild flowers honey », oft die Stufe des Leit- oder Begleitpollens erreicht, drängte sich eine nähere Identifizierung auf. $\mathrm{Zu}$ diesem $\mathrm{Zweck}$ habe ich eine vergleichende Prüfung des Pollens einer Anzahl in Israel vorkommender Arten der Gattung Trifolium L. unternommen.

Von den Arten mit Pollen der Zander'schen T(rifolium)-Form sind in der Literatur bisher beschrieben worden : Trifolium incarnatum L., T. pratense L., $T$. angustifolium L., T. repens L., T. hybridum L. und T. alexandrinum L. (ERDTMaN, 1966 ; Maurizio, 1956 ; Maurizio, Louveaux, 1965 ; Oustuani, 1976 ; Ricciardelli D'Albore, Persano Oddo, 1978 ; Zander, 1935, 1941).

Die Trifolium-Arten sind charakterisiert durch tricolporoide, isopolare, prolat sphäroidale bis subprolate Pollenkörner. Die Exine ist aus Columellen 
aufgebaut, das Tectum trägt eine lockere Netzstruktur (reticulum); ; das Cytoplasma ist feinkörnig (Bezeichnungen nach Beug, 1961 ; Erdtman, 1966 ; MaURIZIO, Louveaux, 1965).

\section{MATERIAL UND METHODIK}

Das verarbeitete Pflanzenmaterial stammt zum Teil aus dem Herbar des Botanischen Instituts der Hebrew University Jerusalem, zum Teil wurde es in der Schweiz und in Israel gesammelt.

Der aus Blüten entnommene Pollen wurde nach der Methode der Internationalen Kommission für Bienenbotanik der IUBS behandelt, d.h. entfettet, in einer Fruktoselösung zum Quellen gebracht und in Glyzeringelatine zu einem Dauerpräparat eingeschlossen (Louveaux, Maurizio, VorwoHL, 1978). Die Pollenmessungen erfolgten mit Hilfe eines Messokulars bei 480facher Vergrösserung.

\section{ERGEBNISSE UND SCHLUSSFOLGERUNGEN}

Der erste Abschnitt enthält die Literaturangaben über die Pollenmasse der 6 bisher beschriebenen Trifolium-Arten (zur Ergänzung sind die Masse von Lotus corniculatus L. beigefügt). Zwischen den Angaben der einzelnen Autoren bestehen daraus berechneten Form-Index (Verhältnis P/E). Zur leichteren Orientierung ist die Tabelle in 4 Abschnitte gegliedert.

Der erste Abschnitt enthält die Literaturangaben über die Pollenmaße der 6 bisher beschriebenen Trifolium-Arten (zur Ergänzung sind die Maße von Lotus corniculatus L. beigefügt). Zwischen den Angaben der einzelnen Autoren bestehen gewisse Unterschiede. So gibt ERDTMAN für Trifolium pratense Werte von $\mathrm{P}=45 \mu, \mathrm{E}=34 \mu$, mit einem $\mathrm{P} / \mathrm{E}-$ Verhältnis von 1,32 an, was einer subprolaten, beinahe prolaten Pollenform entspricht, während bei den übrigen Autoren die Pollenform mit $\mathrm{P}=36,2-38,1 \mu, \mathrm{E}=34,8-39,7 \mu$ und $\mathrm{P} / \mathrm{E}=0,96-1,08$ prolat sphäroidal, in einem Fall sogar knapp oblat sphäroidal ist. Ähnliche, wenn auch weniger auffällige Unterschiede sind bei Trifolium incarnatum vorhanden. Sie können auf Unterschieden der Präparation beruhen (acetolysierte Präparate bei ERDTMAn, Frischpräparate bei den übrigen Autoren) oder auf unterschiedlichem Pflanzenmaterial, da es sich bei den angeführten Trifolium-Arten um Kulturpflanzen handelt mit zahlreichen Varietäten und Zuchtsorten.

Im zweiten Abschnitt sind die Messwerte für 14 in Israel vorkommende Trifolium-Arten zusammengestellt. Die Extreme bilden Trifolium formosum, das sich mit Werten von $\mathrm{P}=45,1$ und $\mathrm{E}=37,3 \mu$ dem Trifolium incarnatum 
TAB. 1. - Pollenmasse einiger Trifolium-Arten

TABL. 1. - Measures of pollens of some species of Trifolium

\begin{tabular}{|c|c|c|c|c|c|}
\hline \multirow{2}{*}{ Art } & \multirow{2}{*}{ Herkunft } & \multirow{2}{*}{$\begin{array}{c}\text { Material } \\
\text { Präparierung }\end{array}$} & \multicolumn{3}{|c|}{ Seitenlage } \\
\hline & & & $\mathbf{P}_{\boldsymbol{\mu}}$ & $\mathrm{E}_{\mu}$ & $P / E$ \\
\hline Trifolium incarnatum $\mathrm{L}$. & ZANDER, 1935 & Frisch & 49,0 & 41,8 & 1,17 \\
\hline Trifolium incarnatum $\mathbf{L}$. & MaURIZIO, 1956 & Frisch & 43,4 & 38,3 & 1,13 \\
\hline Trifolium incarnatum $\mathbf{L}$. & MaURIzIO/ & & & & \\
\hline & Louveaux, 1965 & Frisch & 52,0 & 42,0 & 1,24 \\
\hline Trifolium pratense $\mathbf{L}$. & ZANDER, 1935 & Frisch & 36,2 & 34,8 & 1,04 \\
\hline Trifolium pratense $\mathrm{L}$. & ERDTMAN, 1966 & Acetolys. & 45,0 & 34,0 & 1,32 \\
\hline Trifolium pratense $\mathbf{L}$. & MaUrizio, 1956 & Frisch & 38,1 & 39,7 & 0,96 \\
\hline Trifolium pratense $\mathrm{L}$. & $\begin{array}{l}\text { MaUrizio/ } \\
\text { Louveaux, } 1965\end{array}$ & Frisch & 38,0 & 35,0 & 1,08 \\
\hline Trifolium angustifolium $\mathrm{L}$. & OUSTUANI, 1976 & Frisch & 38,2 & 35,7 & 1,07 \\
\hline Trifolium repens $\mathrm{L}$. & ZANDER, 1935 & Frisch & 28,6 & 26,0 & 1,09 \\
\hline Trifolium repens $\mathrm{L}$. & MAURIZIO, 1956 & Frisch & 26,4 & 25,2 & 1,04 \\
\hline Trifolium repens $\mathrm{L}$. & MaUrizio/ & & & & \\
\hline & LOUVEAUX, 1965 & Frisch & 27,0 & 25,0 & 1,08 \\
\hline Trifolium hybridum L. & ZANDER, 1935 & Frisch & 22,6 & 21,6 & 1,03 \\
\hline Trifolium hybridum $\mathrm{L}$. & Maurizio, 1956 & Frisch & 26,0 & 23,4 & 1,11 \\
\hline Trifolium alexandrinum $\mathbf{L}$. & ZANDER, 1941 & Frisch & 33,0 & 31,8 & 1,04 \\
\hline Lotus corniculatus L. & ZANDER, 1935 & Frisch & 18,4 & 13,0 & 1,41 \\
\hline Lotus corniculatus $\mathbf{L}$. & $\begin{array}{l}\text { MaURIZIO/ } \\
\text { LOUVEaUX, } 1965\end{array}$ & Frisch & 19,0 & 14,0 & 1,35 \\
\hline $\begin{array}{l}\text { Trifolium formosum D'Urv. }= \\
\quad \text { T. dayurum C. }\end{array}$ & ZOHARY Israel & Herbar & 45,1 & 37,3 & 1,21 \\
\hline Trifolium palaestinum Boiss. & ZOHARY Israel & Herbar & 39,5 & 35,0 & 1,13 \\
\hline Trifolium nervulosum Boiss. et Heldr. & MAURIZIO Israel & Frisch & 30,6 & 27,5 & 1,11 \\
\hline dichroanthum & ZOHARY Israe! & Herbar & 30.3 & 23,5 & 1,29 \\
\hline Trifolium clypeatum $\mathbf{L}$. & ZOHARY Israel & Herbar & 26,6 & 22,2 & 1,20 \\
\hline Trifolium purpureum Loisel. & ZOHARY Israel & Herbar & 26,1 & 19,3 & 1,34 \\
\hline Trifolium campestre Schreb. & Maurizio Schweiz & Frisch & 26,1 & 20,5 & 1,27 \\
\hline Trifolium resupinatum $\mathbf{L}$. & ZOHARY Israel & Herbar & 25,3 & 21,8 & 1,16 \\
\hline Trifolium resupinatum $\mathbf{L}$. & Maurizio Schweiz & Frisch & 26,9 & 23,3 & 1,15 \\
\hline Trifolium resupinatum $\mathbf{L}$. & MaUrizio B.R.D. & Frisch & 24,9 & 21,9 & 1,14 \\
\hline Trifolium vavilovi Eig. & ZoHARY Israel & Herbar & 23,3 & 18,8 & 1,24 \\
\hline Trifolium echinatum Bieb. & ZOHARY Israel & Herbar & 23,1 & 18,5 & 1,25 \\
\hline Trifolium cherleri $\mathrm{L}$. & ZOHARY Israel & Herbar & 22,8 & 18,5 & 1,22 \\
\hline Trifolium Berytheum Boiss. et Bl. & ZOHARY Israel & Herbar & 19,5 & 16,2 & 1,20 \\
\hline $\begin{array}{l}\text { Trifolium alexandrinum } \mathrm{L} . \\
\text { Trifolium alexandrinum } \mathrm{L} .\end{array}$ & MaURizio Schweiz & Frisch & 32,7 & 29,5 & 1,11 \\
\hline $\begin{array}{l}\text { var. alexandrinum } \\
\text { Trifolium alexandrinum } \mathbf{L} \text {. }\end{array}$ & Maurizio Israel & Frisch & 35,9 & 28,9 & 1,24 \\
\hline var. serotinum & MaURIzio Israel & Frisch & 30,5 & 26,8 & 1,14 \\
\hline $\begin{array}{l}\text { Trifolium alexandrinum } \mathrm{L} \text {. } \\
\text { var. latidentatum }\end{array}$ & ZOHARY Israel & Herbar & 40,0 & 32,9 & 1,21 \\
\hline Trifolium constantinopolitanum Ser. & ZOHARY Israel & Herbar & 34,0 & 28,5 & 1,19 \\
\hline Trifolium sp. & Maurizio & Noning I & 32,6 & 29,1 & 1,12 \\
\hline Trifolium sp. & Maurizio & Honig 2 & 33,8 & 29,7 & 1,14 \\
\hline Trifolium sp. & MaUrizio & Honig 3 & 33,1 & 29,9 & 1,11 \\
\hline
\end{tabular}




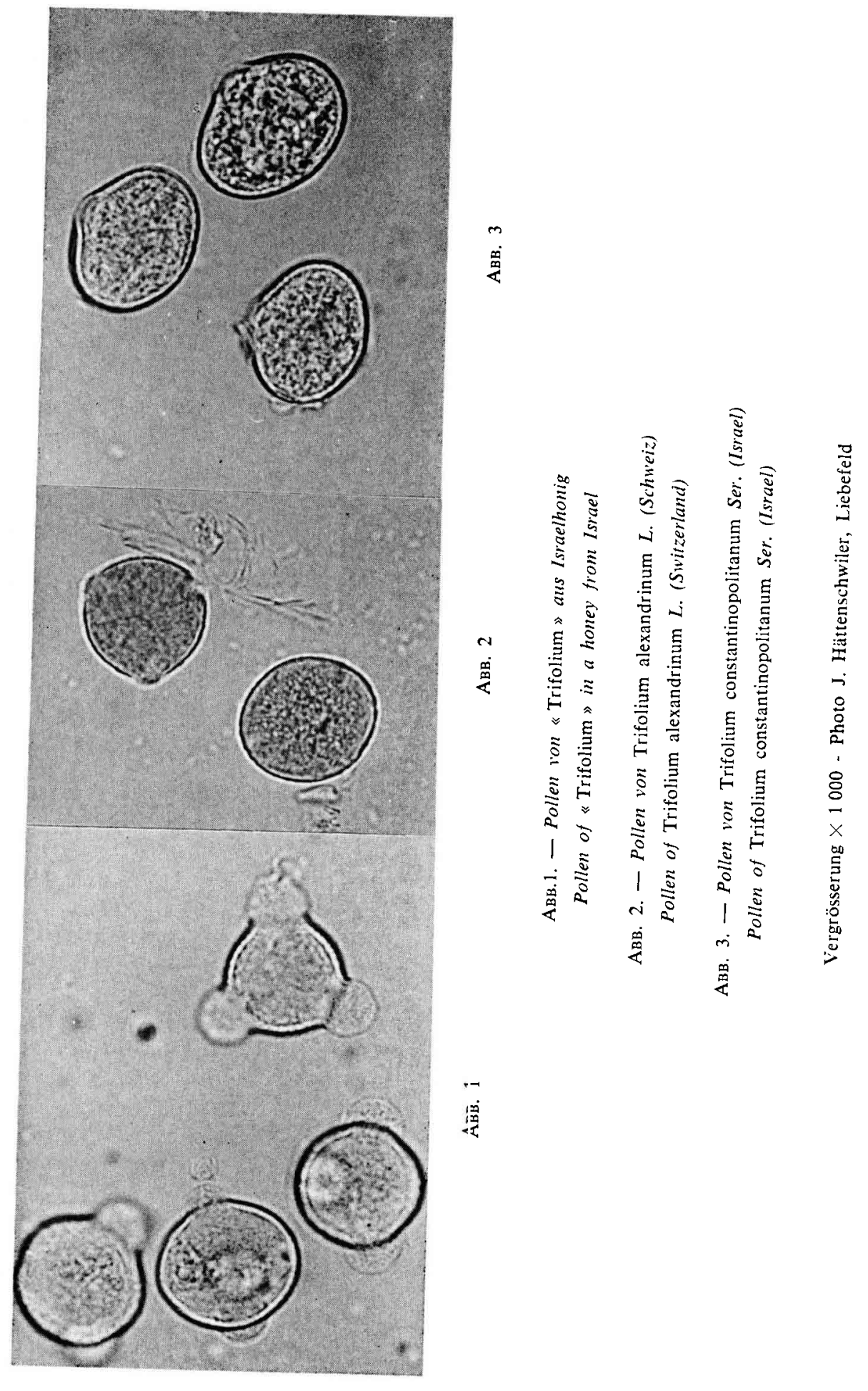


nähert, und Trifolium Berytheum, das mit $P=19,5$ und $E=16,2 \mu$ eher an Kleinformen wie Lotus corniculatus erinnert. Eine Mittelstellung nehmen Trifolium nervulosum und $T$. dichroanthum ein $(P=$ ca $30 \mu)$; die übrigen, $T$. clypeatum, $T$. purpureum, T. campestre, $T$. resupinatum, $T$. vavilovi, T. echinatum, $T$. cherleri, entsprechen mit $\mathrm{P}=$ Werten zwischen 23 und $27 \mu$ der Gruppe von Trifolium repens $/ T$. hybridum. Der Form-Index $\mathrm{P} / \mathrm{E}$ liegt bei 13 Arten zwischen 1,11 und 1,29, was der prolat sphäroidalen bis subprolaten Pollenform entspricht; nur Trifolium purpureum hat mit einem $\mathrm{P} / \mathrm{E}$-Verhältnis von 1,34 knapp prolate Pollenkörner. Auch in bezug auf die Oberflächenstruktur sind unter den 14 Arten Unterschiede vorhanden. Am kräftigsten ist die Netzstruktur bei Trifolium formosum, $T$. clypeatum und $T$. vavilovi, am schwächsten bei $T$. palaestinum, $T$. campestre, $T$. nervulosum, $T$. resupinatum und $T$. Berytheum.

Der folgende Abschnitt zeigt die Messwerte für Trifolium alexandrinum $\mathrm{L}$. in einigen Varietäten, inbegriffen $T$. constantinopolitanum SER., das als identisch oder als Varietät von T. alexandrinum betrachtet wird (Tutin et al., 1968). Von den 5 untersuchten Varietäten von $T$. alexandrinum entsprechen 3 , mit P-Werten von 33-36 $\mu$, annähernd den Angaben von ZANDER (1941); nur var. serotinum hat etwas kleinere $(P=30,5 \mu)$, var. latidentatum deutlich grössere Pollenkörner $(P=40 \mu)$. Es bestehen demnach innerhalb der Art Trifolium alexandrinum Unterschiede der Pollengrösse, was bei Kulturpflanzen oft zu beobachten ist. Das P/E-Verhältnis bleibt mit Werten von 1,11 bis 1,24 im Rahmen der prolat sphäroidalen bis subprolaten Form. Nach Grösse und Form nimmt Trifolium alexandrinum eine Mittelstellung ein zwischen den Gruppen $T$. incarnatum $/ T$. pratense und $T$. repens $/ T$. hybridum.

Bei 4 der untersuchten Formen von Trifolium alexandrinum ist die Netzstruktur der Exine ziemlich undeutlich ausgebildet, was der Beschreibung von ZANDER entspricht. Eine Ausnahme bildet $T$. constantinopolitanum, dessen Pollenkörner eine kräftigere Netzstruktur zeigen (Abb. 2, 3).

Im letzten Abschnitt der Tabelle sind die Messwerte für die «Trifolium》Form aus 3 Israelhonigen enthalten, in welchen sie als Leitpollen auftrat. Die Werte sind untereinander gut ausgeglichen und liegen mit $\mathrm{P}=32,6-33,8 \mu$, $\mathrm{E}=29,1-29,9 \mu$ und $\mathrm{P} / \mathrm{E}=1,11-1,14 \mathrm{im}$ Rahmen der Mehrzahl der Varietäten von Trifolium alexandrinum. Auch in bezug auf die Exinestruktur besteht zwischen der «Trifolium »-Form aus Honig und $T$. alexandrinum gute Übereinstimmung (Abb. 1).

Es ist deshalb anzunehmen, dass der in Israelhonigen so häufig vorhandene Trifolium-Pollen zur Hauptsache von Trifolium alexandrinum stammt, einer in Israel verbreiteten, in mehreren Varietäten kultivierten Pflanze. In Frage kommen ausserdem Trifolium nervulosum und $T$. dichroanthum, alle übrigen bisher untersuchten Trifolium-Arten haben entweder grösseren oder kleineren Pollen. 
Das Pollenspektrum vieler Israelhonige, besonders der als «wild flowers honey » deklarierten, nähert sich dem ägyptischer Honige, die nach RICCIARDELLI D'Albore, VorwoHl (1980) ; VorwoHL (1981) und Zander (1941) oft Trifolium alexandrinum als Leitpollen führen. Ein deutlicher Unterschied besteht dagegen zu Honigen aus dem übrigen Mittelmeergebiet, in welchen Trifolium alexandrinum im allgemeinen eine untergeordnete Rolle $\mathrm{zu}$ spielen scheint (LouvEAux, ABED, 1984 ; Oustuani, 1976 ; Ricciardelli D'Albore, 1980, 1983 ; RiCCiaRdelli D'Albore, VorWOHL, 1980 ; VORWOHL, 1981).

Eingegangen im April 1984.

\section{DANKSAGUNG}

Für Pflanzenmaterial und Auskünfte über die Verbreitung von Trifolium-Arten in Israel bin ich zu grossem Dank verpflichtet den Herren : Prof. Dr. M. Zohary und Dr. D. Heller, Botanisches Institut der Hebrew University Jerusalem, Prof. Dr. GaliL, Botanisches Institut der Universität Tel Aviv, Dr. Y. Lensky, Entomologisches Institut der Hebrew University Rehovot; für Honigproben und Auskünfte den Herren : Ch. Kalman, Gan-Haim und M. sc. J. Linder, Motza-Ilith. Herrn J. Háttenschwiler, Liebefeld verdanke ich die Pollenphotos, Frau O. Rochat, Bex die Französisch-Übersetzung der Zusammenfassung.

\section{RÉSUMÉ \\ DETERMINATION DUN POLLEN DU GROUPE TRIFOLIUM DANS LES MIELS D'ISRAEL}

Le miel d'Israël, surtout le «wild flowers honey» contient souvent un pollen dominant ou d'accompagnement de forme Trifolium dont l'identification causa d'abord quelques difficultés. Un examen comparatif des pollens de 14 espèces de Trifolium qu'on trouve en Israël et de 5 variétés de Trifolium alexandrinum a permis une détermination plus précise de cette forme. Il s'agit de Trifolium alexandrinum L., une espèce répandue en IsraëI, cultivée en plusieurs variétés. Le spectre pollinique de nombreux miels d'Israël se rapproche de celui du miel égyptien dans lequel le pollen de Trifolium alexandrinum est souvent le pollen dominant.

\section{SUMMARY}

\section{IDENTIFICATION OF A POLLEN OF THE TRIFOLIUM-GROUP IN HONEYS FROM ISRAEL}

Honeys from Israel, especially «wild flowers honey», frequently contain a form of Trifolium, not previously identified, as a predominant or secondary pollen. This pollen was compared with that of 14 Trifolium species occuring in Israel and the pollen of 5 varieties of $T$. alexandrinum L., a commonly cultivated plant in Israel. As the result of this the unknown pollen was identified as T. alexandrinum. 


\section{LITERATUR}

Beug H.-J., 1961. - Leitfaden der Pollenbestimmung. G. Fischer, Stuttgart.

Erdtman G., 1966. - Pollen morphology and plant taxonomy. I. Angiosperms. Hafner, New York. Louveaux J., ABed L., 1984. - Les miels d'Afrique du Nord et leur spectre pollinique. Apidologie, 15 (1), 145-170.

Louveaux J., Maurizio A., Vorwohl G., 1978, - Methods of melissopalynology. Bee World, 59, 139-157.

MaUrizio A., 1956. - Pollengestaltung bei einigen polyploiden Kulturpflanzen. Grana Palynol. (N.S.), 1, 59-69.

Maurizio A., 1968. - Das Pollenbild einiger Honige aus Israel. Z. Bienenforsch., 9, $216-217$. Maurizio A., Louveaux J., 1965. - Pollens de plantes mellifères d'Europe. U.G.A.F. Paris.

Oustuani A.M., 1976. - Das mikroskopische Bild der Honige des östlichen Mittelmeergebietes. Diss. Universität Hohenheim.

Ricciardellt D'Albore G.C., 1980. - Contributo alla conoscenza della flora nettarifera del Marocco sulla base dell'analisi microscopica dei mieli. Riv. Agric. Subtrop. Trop. LXXIV, $57-72$.

Ricciardelli D'Albore G.C., 1983. - Problemi relativi alla conoscenza della flora apistica nel Bacino del Mediterraneo. Riv. Agric. Subtrop. Trop. LXXVII, 93-121.

Ricciardelli D'Albore G.C., Persano Oddo L., 1978. - Flora apistica italiana. Ist. Sper. Zool. Agr. Firenze.

Ricciardell D'Albore G.C., Vorwohl G., 1980. - Sortenhonige im Mittelmeergebiet. Riv. Agric. Subtrop. Trop. LXXIV, 89-118.

Tutin T.G. et al. Edit, 1964-1983. - Flora Europaea. Univ. Press. Cambridge.

Vorwohl G., 1981. - Pollen spectra of African honeys. IV. Int. Palyn. Conf., Lucknow (1976-1977), 3, 499-502.

ZaNder E., 1935, 1941. - Pollengestaltung und Herkunftsbestimmung bei Blütenhonig, I, III. Liedloff, Loth, Michaelis, Leipzig.

Zohary M., Feinbrun N., 1948. - Analytical flora of Palestine, sec. Ed. Palestine. J. Bot., Jerusalem. 